\title{
Phase locking between Josephson soliton oscillators
}

Holst, T.; Hansen, Jørn Bindslev; Grønbech-Jensen, N.; Blackburn, J. A.

Published in:

Physical Review B

Link to article, DOI:

10.1103/PhysRevB.42.127

Publication date:

1990

Document Version

Publisher's PDF, also known as Version of record

Link back to DTU Orbit

Citation (APA):

Holst, T., Hansen, J. B., Grønbech-Jensen, N., \& Blackburn, J. A. (1990). Phase locking between Josephson soliton oscillators. Physical Review B, 42(1), 127-131. https://doi.org/10.1103/PhysRevB.42.127

\section{General rights}

Copyright and moral rights for the publications made accessible in the public portal are retained by the authors and/or other copyright owners and it is a condition of accessing publications that users recognise and abide by the legal requirements associated with these rights.

- Users may download and print one copy of any publication from the public portal for the purpose of private study or research.

- You may not further distribute the material or use it for any profit-making activity or commercial gain

- You may freely distribute the URL identifying the publication in the public portal

If you believe that this document breaches copyright please contact us providing details, and we will remove access to the work immediately and investigate your claim. 


\title{
Phase locking between Josephson soliton oscillators
}

\author{
T. Holst, J. Bindslev Hansen, N. Grønbech-Jensen, and J. A. Blackburn* \\ Physics Laboratory I, Modellering Ikke-Lineaer Dynamik og Irreversibel Termodynamik, \\ The Technical University of Denmark, DK-2800 Lyngby, Denmark
}

(Received 19 January 1990)

\begin{abstract}
We report observations of phase-locking phenomena between two Josephson soliton (fluxon) oscillators biased in self-resonant modes. The locking strength was measured as a function of bias conditions. A frequency tunability of the phase-locked oscillators up to $7 \%$ at $10 \mathrm{GHz}$ was observed. Two coupled perturbed sine-Gordon equations were derived from an equivalent circuit consisting of inductively coupled, nonlinear, lossy transmission lines. These equations were solved numerically to find the locking regions. Good qualitative agreement was found between the experimental results and the calculations based on the model.
\end{abstract}

\section{INTRODUCTION}

Over the past two decades phase-locking of nonlinear Josephson junction oscillators has been studied extensively for systems of small junctions, i.e., junction dimensions smaller than $\lambda_{J}$, the Josephson penetration depth. ${ }^{1}$ A Josephson transmission line (JTL), i.e., a long and narrow, quasi-one-dimensional Josephson tunnel junction of length $l$ and width $w$, is a nonlinear device which supports self-resonant solitonic modes. ${ }^{2}$ We report on observations of phase-locking effects between two long Josephson junctions operating in the soliton mode and on the results of numerical simulations of a model of this system consisting of two coupled perturbed sine-Gordon equations. The resonant motion of fluxons and antifluxons induces self-pumped current singularities, the zero-field steps (ZFS's) in the junction dc current-voltage characteristic. They occur at voltages $V= \pm n \bar{c} \Phi_{0} / l$, where $\bar{c}$ is the Swihart velocity of light in the barrier of the junction, $n$ is the number of fluxons or antifluxons, and $\Phi_{0}=h / 2 e$ is the magnetic flux quantum. Phase-locked arrays of soliton oscillators are of technological interest because they offer larger power and narrower linewidth.

Similar phase-locking phenomena have been observed previously by Finnegan and Wahlsten ${ }^{3}$ and by Cirillo and Lloyd. ${ }^{4}$ We present here the first detailed experimental characterization of the phase-locking both at dc and at microwave frequencies, along with the first qualitative comparison between the experiment and simulated dc $I-V$ curves in the locking range.

\section{EXPERIMENTAL TECHNIQUE}

Experiments were carried out on intermediate length $\mathrm{Nb}-\mathrm{Nb}_{x} \mathrm{O}_{y}-\mathrm{Pb}$ tunnel junctions which had typical values of $\quad l=400 \mu \mathrm{m}, \quad w=20 \mu \mathrm{m}, \quad \lambda_{J} \approx 100-250 \mu \mathrm{m}$, $\bar{c} / c_{\text {vacuo }} \approx 0.025$, and a critical current density $J_{c}=0.7 \Delta I_{\text {gap }} / w l$, of $3-16 \mathrm{~A} / \mathrm{cm}^{2}$, where $70 \%$ of the current increase at the gap, $\Delta I_{\text {gap }}$, was used to eliminate effects of the spatial variation of the bias current in the (strong-coupled) junctions. The sample geometry with two JTL's fabricated side by side sharing the $\mathrm{Pb}$ counter electrode is shown in Fig. 1. Four samples with distance $d=35$ or $75 \mu \mathrm{m}$ between the junctions were investigated experimentally. The nominal junction length was designed to give a resonance frequency $\bar{c} / 2 l$ around 10 GHz. As shown in Fig. 1, two independent current sources were used, one providing a common dc bias, $I_{\text {com }}$, the other, $I_{\text {trim }}$, allowing for adjustment of the current through one of the junctions (No. 1). The total voltage across the pair of junctions in series could be monitored.

The sample was screened against external noise sources by electric and magnetic shielding and filtering. It was placed in a vacuum can immersed in a pressure-regulated helium bath at $4.2 \mathrm{~K}$. A microwave coupling to the sample was established by a coaxial cable which was connected to the $50-\Omega \mathrm{Nb}$ microstrip line which also formed the bottom electrode of the junctions. The microwave signal generated in the junctions was coupled out through the microstrip connection to a microwave detection system including a spectrum analyzer.

\section{EXPERIMENTAL RESULTS}

The $I_{\text {com }}$ versus $V$ curve of two series-connected JTL's exhibited two distinct critical currents, as well as the ZFS's of the solitonic fluxon modes of the two junctions individually and in combination of the ZFS's. By the application of the trimming current $I_{\text {trim }}$ it was possible to make the junction voltages $V_{1}$ and $V_{2}$ coincide for a given value of $I_{\text {com }}$. Distinct voltage-locking regions were then observed in the $I_{\text {com }}$ versus $V_{1}+V_{2}$ characteristic, see Fig. 2. The range over which the common bias current could be varied (at fixed $I_{\text {trim }}$ ) without losing phase lock, is a measure of the strength of the phase locking between the $(\mathrm{ZFS}+1)-(\mathrm{ZFS}+1)$ or $(\mathrm{ZFS}+1)$ $(\mathrm{ZFS}-1)$, corresponding to the two current configurations: series aiding $(\rightarrow \rightarrow)$ or series opposed $(\rightarrow \leftarrow)$. Varying $I_{\text {trim }}$ caused the locking range to move from the bottom towards the top of the ZFS. In Fig. 3 we have plotted the measured normalized locking range versus $I_{\text {trım }} / \bar{I}_{c}$ for both current configurations. $\bar{I}_{c}$ denotes the average critical current $\left(I_{c 1}+I_{c 2}\right) / 2$, where 


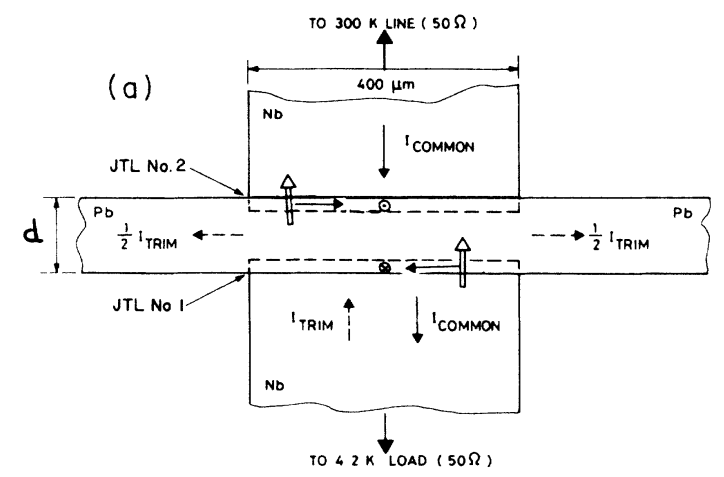

(b)

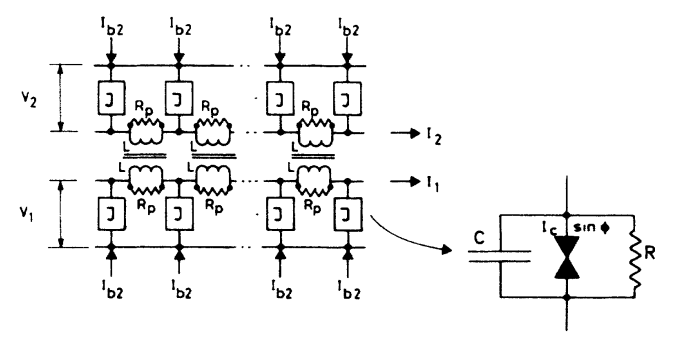

FIG. 1. (a) Sample geometry and dc bias arrangement for two Josephson transmission lines (JTL's). The Lorentz force on flux quanta (open arrows) in the junctions is indicated. (b) Equivalent circuit used to model the pair of inductively coupled JTL's.
$I_{c 1,2}=J_{c 1,2} w l$, and $\Delta=\left|I_{c 1}-I_{c 2}\right| / \bar{I}_{c}$. Measured in this way the locking strength is greater for the series aiding configuration because in that case the voltage pulling (i.e., frequency pulling) necessary to achieve phase locking is smaller due to the nearly identical slopes of the two ZFS's in the region of locking. For the series opposed case the two ZFS's have slopes of opposite signs (for varying $\left.I_{\text {com }}\right)$. For two of the samples, $A$ with $d=75 \mu \mathrm{m}$ and $B$ with $d=35 \mu \mathrm{m}$, the ratio of the locking range for the series opposed configuration was 0.4 (comparable values of $\Delta \approx 0.30$ ).

The interaction between the two soliton oscillators was also observed in the detected microwave signals as phase locking, mixing, and linewidth narrowing. The insets in the lower part of Fig. 2 reproduce some of the microwave spectra from the two junctions both individually and in combination showing phase locking. A maximum power output is expected to be $P_{12}=\left(\sqrt{P_{1}}+\sqrt{P_{2}}\right)^{2}$ for two phase-locked oscillators (note that there was a large impedance mismatch between the $50-\Omega$ microwave circuit and the low-impedance JTL's). For both current configurations we observed a surprisingly strong enhancement of the power detected in the coherent state of up to $2.0 P_{12}$ around the middle of the locking range. A narrowing in the linewidth was often observed for the locked oscillators (cf. spectra $A, B$, and $C$ in Fig. 2). On the top of the ZFS where the linewidth is below $50 \mathrm{kHz}$, which is of technical interest, a linewidth down to $4 \mathrm{kHz}$ was observed. A frequency tunability around 1-2\% was

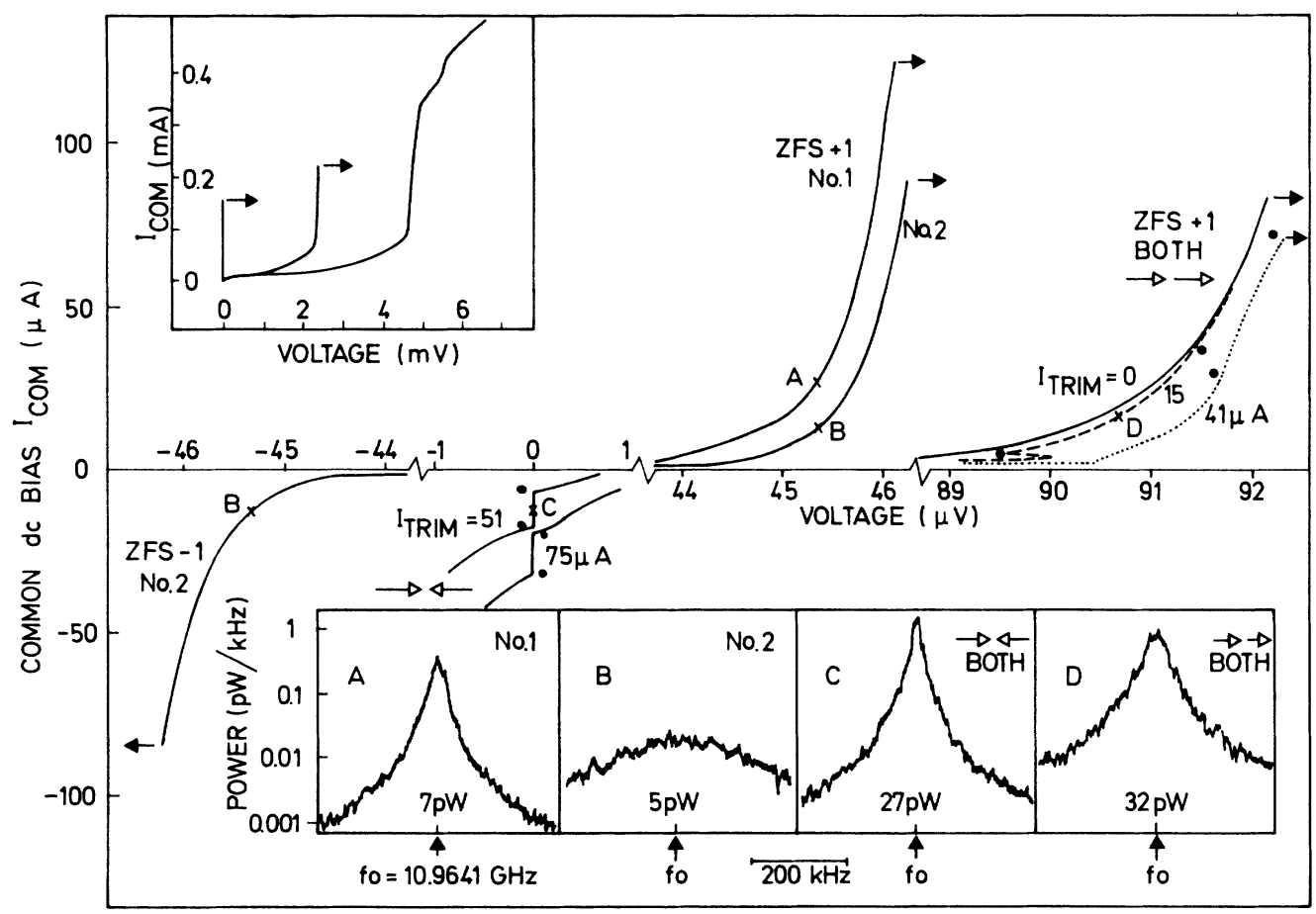

FIG. 2. Measured $I_{\mathrm{com}}-V$ characteristics and microwave radiation spectra showing the phase-locking phenomena in a pair of JTL's, sample $B(d=35 \mu \mathrm{m})$. The inset in the upper left corner displays the overall $I-V$ characteristic. Detailed $I_{\text {com }}$ vs $V_{1}+V_{2}$ curves are shown for the $(Z F S+1)-(Z F S+1)$ configuration [series aiding $(\rightarrow \rightarrow)$ ] and for the $(Z F S+1)-(Z F S-1)$ configuration [series opposed $(\rightarrow \leftarrow)$ ]. The locking ranges are indicated by dots $(\bullet)$ for various values of the trim current $I_{\text {trim }}$. The microwave radiation spectra in the lower insets were detected at the bias points indicated by $A, B, C$, and $D$. The integrated detected power is given for each spectrum. 


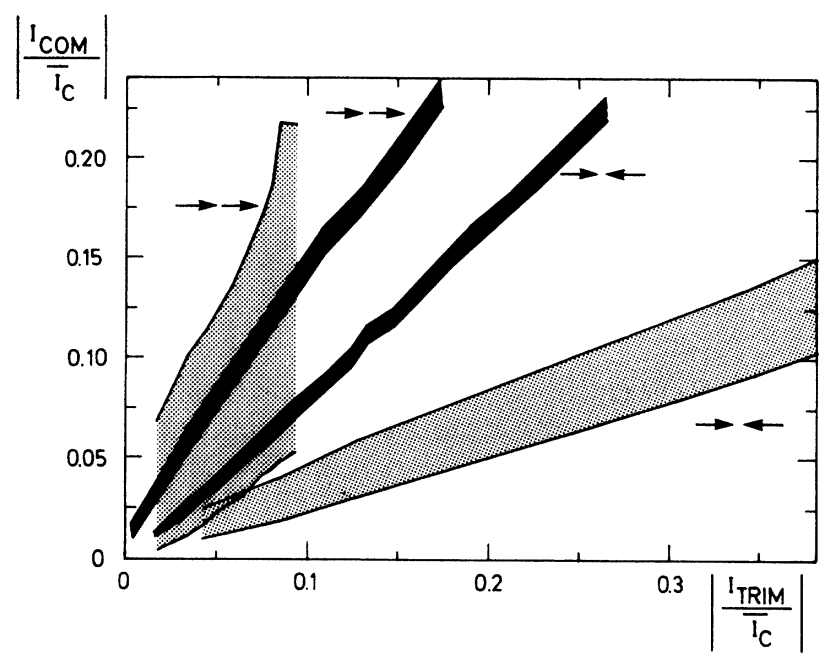

FIG. 3. Experimental normalized locking range versus the normalized trim current on the ZFS's for both current configurations: series aided $(\rightarrow \rightarrow)$ and series opposed $(\rightarrow \leftarrow)$. The black area indicates the locking range for sample $A, I_{c 1}<I_{c 2}$, and the shaded area for sample $B, I_{c 1}>I_{c 2}$. Sample parameters were $\bar{I}_{c}(A)=1007 \mu \mathrm{A}, \quad \bar{I}_{c}(B)=259 \mu \mathrm{A}, \quad \Delta(A)$ $=0.31, \Delta(B)=0.30, l / \lambda_{J}(A)=3.4$, and $l / \lambda_{J}(B)=1.7$.

typically achieved. Phase locking was also achieved for the $(Z F S+1)-(Z F S+2)$ and $(Z F S+2)-(Z F S+2)$ modes. Measurements on a sample with nearly identical junctions $(\Delta \approx 0.05)$ indicated the capability of phase locking with no trimming current and a frequency tunability up to $7 \%$. The typical frequency range of phase locking (for a fixed value of $I_{\text {trim }}$ ) of $100-200 \mathrm{MHz}$ was typically 100 times larger than previously observed for two coupled soliton oscillators. ${ }^{3,4}$ The linewidth and power values were of the same order of magnitude as in these reports $\left[\Delta \omega \approx(2 \pi) 10 \mathrm{kHz}, P_{12} \approx 20 \mathrm{pW}\right]$.

\section{THEORETICAL MODEL}

When modeling the coupled soliton oscillators we represent the interaction in the form of a distributed mutual inductance between two resistor-capacitor shunted junctions as shown in Fig. 1. This model is plausible be- cause the fluxon oscillators are operating side by side in close proximity to one another and because the sample geometry utilizes a common connecting electrode. A similar approach was taken in Ref. 5 to model two JTL's vertically stacked one above the other and sharing a common center electrode, although in that system only one JTL was operating as a fluxon oscillator (the second served as a radiation detector), and hence no locking phenomena were involved. Since our sample geometry places the JTL's side by side, the magnitude of the mutual inductance will likely be much less than the value estimated $(M / L=0.28$ ) for the stacked geometry. Nevertheless, we shall find that locking arises even for small values of $M / L$.

The equivalent circuit for two coupled JTL's is shown in Fig. 1. Each line is comprised of lumped Josephson elements (per unit length) characterized by a critical current $I_{c}$, shunt capacitance $C$, and leakage resistance $R$. In addition, each line has linking inductances $L$ (per unit length) and resistances (per unit length) $R_{p}$ which account for energy storage and quasiparticle scattering, respectively, in the superconducting films. The JTL's are coupled by mutual inductances $M$ (per unit length), as indicated.

The equations which govern the voltage and current in JTL No. 1 are

$$
\begin{gathered}
\frac{\partial V_{1}}{\partial x}=-L\left(\frac{\partial I_{1}}{\partial t}-\frac{1}{R_{p}} \frac{\partial}{\partial t} \frac{\partial V_{1}}{\partial x}\right) \\
+M\left(\frac{\partial I_{2}}{\partial t}+\frac{1}{R_{p}} \frac{\partial}{\partial t} \frac{\partial V_{2}}{\partial x}\right), \\
\frac{\partial I_{1}}{\partial x}=I_{b 1}-C \frac{\partial V_{1}}{\partial t}-I_{c 1} \sin \left(\phi_{1}\right)-\frac{V_{1}}{R},
\end{gathered}
$$

where the subscripts $(1,2)$ identify the transmission line with which a given variable is associated, $\phi$ is the Josephson phase, and $I_{b}$ is the bias current per unit length in the JTL. A similar pair of expressions may be written for $\partial V_{2} / \partial x$ and $\partial I_{2} / \partial x$. Combining Eqs. (1) and (2), and employing $V_{1,2}=(\hbar / 2 e)\left(\partial \phi_{1,2} / \partial t\right)$, we obtain

$$
\frac{\hbar}{2 e} \phi_{1 x x}=L\left(\frac{\hbar C}{2 e} \phi_{1 t t}+I_{c 1} \sin \left(\phi_{1}\right)+\frac{\hbar}{2 e R} \phi_{1 t}-\frac{\hbar}{2 e R_{p}} \phi_{1 x x t}-I_{b 1}\right)-M\left(\frac{\hbar C}{2 e} \phi_{2 t t}+I_{c 2} \sin \left(\phi_{2}\right)+\frac{\hbar}{2 e R} \phi_{2 t}-\frac{\hbar}{2 e R_{p}} \phi_{2 x x t}-I_{b 2}\right)
$$

and similarly for $(\hbar / 2 e) \phi_{2 x x}$. Because the critical currents $I_{c 1}$ and $I_{c 2}$ are taken to be unequal, it will be convenient to define as $\lambda_{J}=\left(\Phi_{0} l / 2 \pi L \bar{I}_{c}\right)^{1 / 2}$ and as the plasma frequency $\omega_{0}=\left(2 \pi \bar{I}_{c} / \Phi_{0} C\right)^{1 / 2}$.

The differential equations may be recast in dimensionless form by expressing time in units of $\omega_{0}^{-1}$ and distance in units of $\lambda_{J}$. The normalized parameters are $\alpha=\left(\omega_{0} R C\right)^{-1}, \beta=\omega_{0} L / R_{p}$, and $\delta=M / L$. It is also useful to define a new pair of phase coordinates $\theta \equiv \phi_{1}+\phi_{2}$ and $\Psi \equiv \phi_{1}-\phi_{2}$. From Eq. (3) and its counterpart for $\phi_{2 x x}$, it is possible to derive the following normalized, coupled equations: 


$$
\begin{aligned}
& \theta_{x x}=(1-\delta)\left[\theta_{t t}+\alpha \theta_{t}-\beta \theta_{x x t}-\frac{I_{b 1}+I_{b 2}}{\bar{I}_{c}}+2 \sin \left(\frac{\theta}{2}\right) \cos \left(\frac{\psi}{2}\right)+\Delta \cos \left(\frac{\theta}{2}\right) \sin \left(\frac{\psi}{2}\right)\right], \\
& \psi_{x x}=(1+\delta)\left[\psi_{t t}+\alpha \psi_{t}-\beta \psi_{x x t}-\frac{I_{b 1}-I_{b 2}}{\bar{I}_{c}}+\Delta \sin \left(\frac{\theta}{2}\right) \cos \left(\frac{\psi}{2}\right)+2 \cos \left(\frac{\theta}{2}\right) \sin \left(\frac{\psi}{2}\right)\right]
\end{aligned}
$$

Equations (4) and (5) represent the final form of the description of the coupled Josephson transmission lines. In the absence of an applied magnetic field the boundary conditions for the overlap JTL geometry are given by $\theta_{x}(0, t)=\theta_{x}\left(l / \lambda_{J}, t\right)=0$ and $\psi_{x}(0, t)=\psi_{x}\left(l / \lambda_{J}, t\right)=0$. In Refs. 6 and 7 the interactions between a pair of coupled long Josephson junctions were modeled in this manner, although the authors did not explicitly consider phase locking.

\section{NUMERICAL SIMULATIONS}

Numerical solutions of Eqs. (4) and (5) were obtained using an implicit finite-difference scheme described in Ref. 8. The two sets of tridiagonal equations which arise at each time step was solved by means of an algorithm discussed in Ref. 9. Generally speaking, a time grid of 0.02 of $\omega_{0}$ and a space grid of 0.01 of the total normalized length $l / \lambda_{J}$ were used. At each time step, the position of either soliton was determined by finding the maximum in the spatial distribution of the numerical value of the voltage $\left|\phi_{t}\right|$. From the position versus time data it was then possible to calculate successive periods for the solitons. In many instances the periods exhibited self modulation and, consequently, meaningful results could be obtained only by averaging over ten's of thousands of time steps. Even so such low-frequency mixing products made the numerical averaging less precise at the boundary of the locking region.

Using the inductive coupling model, we have carried out extensive numerical simulations of the phase-locking phenomenon in two coupled JTL's. The locking range was investigated for fixed (typical) values of the junction parameters $l / \lambda_{J}=3, \alpha=0.05, \beta=0.02$, and $\Delta=0.30$ while $\delta$ was varied over the range 0.005 to 0.03 . The sign of $\delta$ was chosen in accordance with the interaction between two magnetic dipoles. In accordance with the experimental configuration, the simulation included a common bias current and a trim current source (see Fig. 1). Figure 4 shows the results of simulations for $\delta=0.0075$ for both current configurations. The normalized locking ranges are seen to be 0.035 and 0.15 for the series opposed and series aiding case, respectively. Qualitatively the same asymmetry in the locking range was observed experimentally: 0.038 and 0.20 for the series opposed and series aiding case, respectively. Good qualitative agreement between experiment and model was also found in the high-frequency spectra around the locking range, in both cases strong frequency pulling and mixing phenomena were seen. We conclude that the coupled transmission line model captures the essence of the phase-locking be- havior. Refinements of the model to account for the details of the observed $I-V$ curves in the locking range could include a more realistic bias current distribution (peaked at the thin-film edges) as well as slight differences in the junction capacitances (different $\bar{c}$ for the junctions, for samples $A$ and $B$ the relative difference was $1.3 \%$ and $0.3 \%$, respectively).
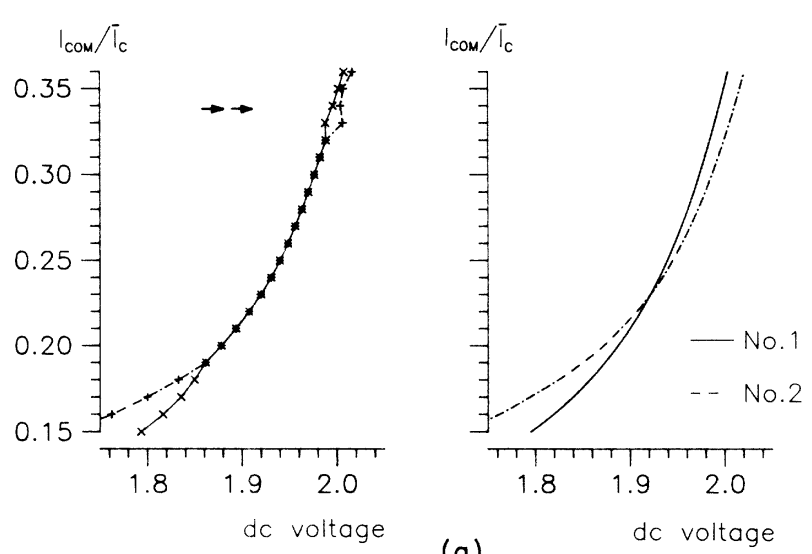

(a)

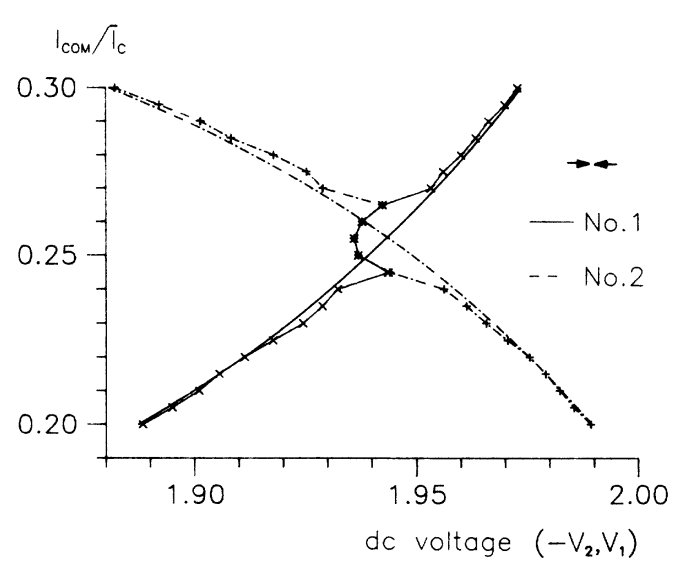

(b)

FIG. 4. I-V curves based on numerical calculations. (a) and (b) show the individual $I-V$ curves for the two junctions in the series aiding and the series opposed case, respectively. Markers show the results of two interacting solitons, belonging to different transmission lines. Straight lines are drawn for clarity. The $I-V$ curves without markers are for the ZFS +1 state in one junction and no soliton present in the other junction. $\alpha=0.05$, $\beta=0.02, \Delta=0.3, l / \lambda_{J}=3, \delta=0.0075$, and $I_{\text {trim }} / \bar{I}_{c}=0.06$ and 0.52 for the series aiding and the series opposed configurations, respectively. Voltage is normalized to $\hbar \omega_{0} / 2 e$. 


\section{CONCLUSIONS}

We have demonstrated new strong phase-locking phenomena between two closely spaced fluxon oscillators based on intermediate length Josephson transmission lines. Phase locking was seen both in the dc $I-V$ characteristics and in the high-frequency radiation. The development of coherent microwave sources consisting of many Josephson junctions is of technological interest. ${ }^{10}$ In this respect, the combination of a common bias and a trim current source (one for each junction) is found to be advantageous with respect to maximizing the locking range and the series opposed current configuration is found to produce a substantial linewidth narrowing. We estimate that 40 coherent oscillators of this type would suffice to generate sufficient power to be useful as a local oscillator in an SIS mixer (about $1 \mu \mathrm{W}$ at $100 \mathrm{GHz}$, since
$P_{N} \sim N^{2} \omega^{2} P_{1}$ for $N$ junctions at frequency $\left.\omega\right)$.

We have modeled the system as two inductively coupled Josephson transmission lines. This leads to two coupled perturbed sine-Gordon equations which we have solved numerically. Agreement between numerical results and experimental observations is good.

\section{ACKNOWLEDGMENTS}

We thank M. R. Samuelsen, M. Salerno, M. Devoret, and N. F. Pedersen for fruitful discussions, G. Friis Eriksen for technical assistance, and I. Rasmussen and S. Hjorth for the sample fabrication. This work was partly supported by the Danish Natural Science Research Council, The Hartmann Foundation, The Danish Research Academy, and the Natural Sciences and Engineering Research Council of Canada.
"Permanent address: Department of Physics and Computing, Wilfrid Laurier University, Waterloo, Ontario, Canada N2L 3 C5.

${ }^{1}$ J. Bindslev Hansen and P. E. Lindelof, Rev. Mod. Phys. 56, 431 (1984); A. K. Jain, K. K. Likharev, J. E. Lukens, and J. E. Sauvageau, Phys. Rep. 109, 309 (1984).

${ }^{2}$ T. F. Fulton and R. C. Dynes, Solid State Commun. 12, 57 (1973); D. W. McLaughlin and A. C. Scott, Phys. Rev. A 18, 1652 (1978); E. Joergensen, V. P. Koshelets, R. Monaco, J. Mygind, M. R. Samuelsen, and M. Salerno, Phys. Rev. Lett. 49, 1093 (1982); O. A. Levring, N. F. Pedersen, and M. R. Samuelsen, J. Appl. Phys. 54, 987 (1983).

${ }^{3}$ T. F. Finnegan and S. Wahlsten, Appl. Phys. Lett. 21, 541 (1972).

${ }^{4}$ M. Cirillo and F. L. Lloyd, J. Appl. Phys. 61, 2581 (1987); M. Cirillo, A. R. Bishop, and P. S. Lomdahl, Phys. Rev. B 39,
4804 (1989).

${ }^{5}$ J. Qin, K. Enpuku, and K. Yoshida, J. Appl. Phys. 63, 1130 (1988).

${ }^{6}$ Y. S. Kivshar and B. A. Malomed, Phys. Rev. B 37, 9325 (1988).

${ }^{7}$ M. B. Mineev, G. S. Mkrtchyan, and V. V. Schmidt, J. Low Temp. Phys. 45, 497 (1981).

${ }^{8}$ P. S. Lomdahl, O. H. Soerensen, and P. L. Christiansen, Phys. Rev. B 25, 5737 (1982).

${ }^{9}$ D. M. Young and R. T. Gregory, A Survey of Numerical Mathematics (Addison-Wesley, New York, 1973), Vol. II, p. 586.

${ }^{10}$ T. Nagatsuma, E. Enpuku, F. Irie, and K. Yoshida, J. Appl. Phys. 54, 3302 (1983); K. L. Wan, A. K. Jain, and J. E. Lukens, IEEE Trans. Mag. MAG-25, 1076 (1989); S. Pagano, R. Monaco, and G. Costabile, ibid. MAG-25, 1080 (1989). 


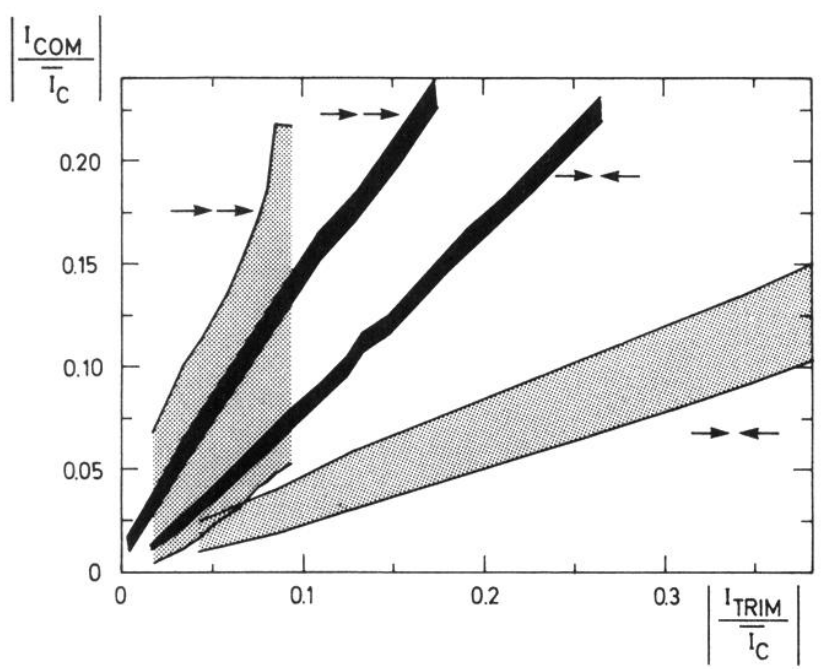

FIG. 3. Experimental normalized locking range versus the normalized trim current on the ZFS's for both current configurations: series aided $(\rightarrow \rightarrow)$ and series opposed $(\rightarrow \leftarrow)$. The black area indicates the locking range for sample $A, I_{c 1}<I_{c 2}$, and the shaded area for sample $B, I_{c 1}>I_{c 2}$. Sample parameters were $\bar{I}_{c}(A)=1007 \mu \mathrm{A}, \bar{I}_{c}(B)=259 \mu \mathrm{A}, \Delta(A)$ $=0.31, \Delta(B)=0.30, l / \lambda_{J}(A)=3.4$, and $l / \lambda_{J}(B)=1.7$. 